\title{
3D electron diffraction: a dedicated device for structural elucidation of nanocrystalline particles
}

\author{
G. Santiso-Quinones, E. Hovestreydt, A. E. Lanza, G. Steinfeld \\ ELDICO Scientific AG, 5234 Villigen, Switzerland \\ santiso@eldico.ch
}

3D Electron Diffraction (3D ED) is a very powerful tool for the structural elucidation of nanocrystalline particles. After its Science nomination for "Breakthrough of the year 2018" [1], 3D ED, using the continuous rotation method [2-3], and well-established crystallographic software, is gaining a lot of attention in all areas of research. In the recent years, many achievements using electron diffraction techniques have been made in the fields of organic and inorganic molecules, polymorphism, geological sciences, natural products, biomolecules, material sciences, energetic materials, batteries, and many others [2-4]. Such experiments are currently done in a (modified) transmission electron microscope, thus requiring customized experimental and data-analysis protocols, which vary depending on each specific instrumental setup. Hence, 3D ED experiments are currently carried out only by specialized staff and require a remarkable investment in terms of time, expertise, knowhow transfer and resources.

A strong need has emerged in the crystallographic community for instrumentation specifically dedicated to 3D ED experiments.

Here we present an electron diffractometer: a new device developed and optimized exclusively for 3D ED which allows a timeeffective, automated, and standardized experimental workflow along with user-friendly operability. Furthermore, the electron diffractometer is conceived to make use exclusively of well-established crystallographic approaches and to interact seamlessly with readily available crystallographic software. Experimental examples of different kind of materials measured with this device will be showcased.

\section{[1] https://vis.sciencemag.org/breakthrough2018/finalists/\#rapid-structure}

[2] Gruene, T., Wennmacher, J. T. C., Zaubitzer, C., Holstein, J. J., Heidler, J., Fecteau-Lefebvre, A., De Carlo, S., Müller, E., Goldie, K. N., Regeni, I., Li, T., Santiso-Quinones, G., Steinfeld, G., Handschin, S., van Genderen, E., van Bokhoven, J. A., Clever, G. H. \& Pantelic, R. (2018) Angew. Chem. Int. Ed. 57, 16313.

[3] Jones, C.G., Martynowycz, M. W., Hattne, J., Fulton, T. J., Stoltz, B. M., Rodriguez, J. A., Nelson, H. M. \& Gonen, T. (2018) ACS Cent. Sci. 4, 1587.

[4] Personal selected list of some achievements: a) Brázda, P., Palatinus, L. \& Babor, M. (2019) Science 364, 667. b) Bücker, R., Hogan-Lamarre, P., Mehrabi, P., Schulz, E. C., Bultema, L. A. Gevorkov, Y., Brehm, W., Yefanov, O., Oberthür, D., Kassier, G. H. \& Miller, R. J. D (2020) Nat. Commun. 11, 996. c) Broadhurst, E. T., Xu, H., Clabbers, M. T. B., Lightowler, M., Nudelman, F., Zou X. \& Parsons, S. (2020) IUCrJ 7, 5. d) Mugnaioli, E., Lanza, A. E., Bortolozzi, G., Righi, L., Merlini, M., Cappello, V., Marini, L., Athanassiou, A. \& Gemmi, M. (2020) ACS Cent. Sci. 6, 1578. e) Kaiukov, R., Almeida, G., Marras, S. Dang, Z., Baranov, D., Petralanda, U., Infante, I., Mugnaioli, E., Griesi, A., De Trizio, L., Gemmi, M. \& Manna, L. (2020) Inorg. Chem. 59, 548. f) Nowroozi, M. A., Wissel, K., Donzelli, M., Hosseinpourkahvaz, N., Plana-Ruiz, S., Kolb, U., Schoch, R., Bauer, M., Malik, A. M., Rohrer, J., Ivlev, S. Kraus, F. \& Clemens, O. (2020) Commun. Mater. 1, 27. g) Fritz, F., Greshake, A., Klementova, M., Wirth, R., Palatinus, L., Trønnes, R. G., Assis Fernandes, V., Böttger, U. \& Ferrière, L. (2020) Am. Mineral. 105, 1704.

Keywords: 3D ED; electron diffraction; nano-crystallography; electron diffractometer 